\title{
Prevalence of Ampc $\beta$-Lactamase-producing Pseudomonas Aeruginosa isolates from feacal matter of cow
}

\begin{abstract}
The possible contamination of food and/or food-producing animals with multidrug resistant bacteria including AmpC-producing Pseudomonas aeruginosa is considered a potential source for the wide dissemination of AmpC $\beta$-lactamase in the community. This portends public health risks for the populace - owing to the multidrug resistant nature of these organisms. There is paucity of data on the prevalence of AmpC-producing bacteria in Abakaliki, Nigeria - which was why this study was carried out. A total of 40 feacal swab samples from cow dung in a local abattoir were bacteriologically examined for the isolation and antimicrobial susceptibility testing of $P$. aeruginosa isolates using standard microbiological procedures on cetrimide selective agar and Kirby-Bauer disk diffusion method respectively. AmpC $\beta$-lactamase was phenotypically confirmed using the ceftazidime-imipenem antagonism test (CIAT). A total of 12 P. aeruginosa isolates were recovered from the samples; and they showed varied levels of resistance to the tested antibiotics especially to cefoxitin, ertapenem, oxacillin, amikacin and cefotaxime. Among the 12 P. aeruginosa isolates, AmpC $\beta$-lactamase was phenotypically detected in $3(25 \%)$ isolates by the CIAT method. This study has presumptively shown that AmpC $\beta$-lactamaseproducing $P$. aeruginosa isolates occur in abattoir. Thus, molecular characterization of the genes that encode AmpC $\beta$-lactamase production in this organism is crucial for a reliable epidemiological investigation into the possible emergence and dissemination of AmpC positive bacteria in the community.
\end{abstract}

Keywords: AmpC $\beta$-lactamase, gram negative bacteria, community acquired infections, abattoir, Nigeria
Volume 4 Issue 5 - 2017

\author{
Chika Ejikeugwu,' Carissa Duru, ${ }^{2}$ Chijioke \\ Edeh,' Ifeanyichukwu Iroha' \\ 'Department of Applied Microbiology, Ebonyi State University, \\ Nigeria \\ ${ }^{2}$ Department of Pharmaceutical Microbiology and \\ Biotechnology, Nnamdi Azikiwe University, Nigeria
}

\begin{abstract}
Correspondence: Ejikeugwu Chika, Department of Applied Microbiology, Faculty of Sciences, Ebonyi State University, Abakaliki, P.M.B 053, Ebonyi State, Nigeria, Tel 2348097684562,
\end{abstract} Email ejikeugwu_chika@yahoo.com

Received: February 02, 2017 | Published: April 13, 2017

\section{Introduction}

Environmentalmicroorganisms represent themostrelevantreservoir of resistance to antibiotics in the community, and this is due in part to the undue exposure of these organisms to antimicrobial agents. AmpC $\beta$-lactamases are clinically important cephalosporinases encoded on the chromosomes of bacteria in the Enterobacteriaceae family and some non-enteric bacteria including Pseudomonas aeruginosa, where they stimulate these organisms to be resistant to the cephamycins (cefoxitin for example). ${ }^{1,2}$ Gram negative bacteria that produce AmpC $\beta$-lactamases have been implicated in many cases of hospital and community acquired infection. ${ }^{1-3}$ For enteric organisms with the potential for high-level AmpC $\beta$-lactamase production by mutation, the development of resistance upon therapy especially to the cephamycins is a concern for public health since these agents are critical in the treatment and management of bacterial-related infections in humans. ${ }^{4}$

AmpC $\quad \beta$-lactamase production in most Enterobacteriaceae including Klebsiella pneumoniae and Escherichia coli is low but the hyper-production of AmpC enzymes in these organisms is usually induced by the exposure of the bacteria to antibiotics. ${ }^{3}$ AmpC $\beta$-lactamases can be expressed at high levels by mutation. The over expression of AmpC $\beta$-lactamases confers resistance to broad-spectrum cephalosporins including cefotaxime, ceftazidime, and ceftriaxone. It has been reported that transmissible plasmids have acquired genes for AmpC $\beta$-lactamases, which consequently can now appear in bacteria lacking or poorly expressing a chromosomal AmpC $\beta$-lactamase gene as is seen in E. coli, K. pneumoniae, and Proteus mirabilis. ${ }^{5}$ The genes that confer or mediate the production of AmpC $\beta$-lactamases in Gram- negative bacteria are often chromosomally-borne rather than being plasmid encoded but these genes may still be plasmid-encoded in some bacteria. ${ }^{6}$ The AmpC $\beta$-lactamases are clinically important betalactamases because they confer antimicrobial resistance to the narrowspectrum, expanded-spectrum and the broad-spectrum cephalosporins and the penicillins; and their resistance is also expressed towards the $\beta$ lactamase inhibitors such as amoxycillin-clavulanic acid. ${ }^{7,8}$ Techniques to identify AmpC $\beta$-lactamase-producing isolates are available but are still evolving and are not yet optimized for the clinical laboratory, which probably now underestimates this resistance mechanism in either the community or hospital environment. Nevertheless, the timely and accurate detection of organisms harbouring genes responsible for the production of AmpC $\beta$-lactamases is critical for total patient care. The occurrence of AmpC-producing P. aeruginosa from abattoir calls for effective monitoring and possibly a ban on the use of antibiotics in the production and/or rearing of food producing animals in order to assuage the emergence and spread of drug resistant bacteria in the community. ${ }^{7,19,21}$ Thus, this study presumptively detected the occurrence of AmpC-producing P. aeruginosa isolates from feacal matter of cow in a local abattoir.

\section{Materials and methods}

\section{Sample collection and processing}

A total of forty (40) swab samples were collected from feacal matter of cow in a local abattoir using sterile swab sticks. After collection, each of the swab sticks was returned into their respective containers, labeled and transported to the Microbiology Laboratory Unit of Ebonyi State University, Abakaliki for bacteriological analysis. 


\section{Isolation and identification of Pseudomonas aeruginosa}

Cetrimide selective agar (Oxoid, UK) containing $10 \mathrm{ml}$ of glycerol was used for the selective isolation of $P$. aeruginosa from the test samples. Each of the feacal swab samples was inoculated in $5 \mathrm{ml}$ double strength of freshly prepared nutrient broth (Oxoid, UK) and incubated for $18-24$ hours at $30 \mathrm{oC}$. A loopful of the broth culture was aseptically streaked on cetrimide selective agar plates; and the plates were incubated at $30 \mathrm{oC} 18-24$ hours. Suspected colonies were inoculated onto freshly prepared cetrimide selective agar for the isolation of pure cultures of $P$. aeruginosa. P. aeruginosa produces greenish/bluish pigmentation on cetrimide selective agar. The suspected $P$. aeruginosa isolates were identified using standard microbiological identification techniques. ${ }^{9,10}$

\section{Susceptibility studies}

Antimicrobial susceptibility testing was carried out using the Kirby-Bauer disk diffusion method as per the guidelines of the Clinical Laboratory Standard Institute (CLSI) with single antibiotic disks comprising (disk concentration in $\mu \mathrm{g}$ ): ceftazidime (CAZ; 30 $\mu \mathrm{g}$ ), gentamicin $(\mathrm{CN} ; 10 \mu \mathrm{g}$ ), cefoxitin (FOX; $30 \mu \mathrm{g}$ ), ciprofloxacin (CIP; $10 \mu \mathrm{g}$ ), ertapenem (ETP; $10 \mu \mathrm{g})$, meropenem (MEM; $10 \mu \mathrm{g}$ ), ceftriaxone (CRO; $30 \mu \mathrm{g})$, cefotaxime (CTX; $30 \mu \mathrm{g})$, ofloxacin (OFX; $\mu \mathrm{g}$ ), amikacin (AK; $10 \mu \mathrm{g}$ ) and imipenem (IMP; $10 \mu \mathrm{g}$ ).Oxoid, UK. Each disk was aseptically placed on Mueller-Hinton agar plates containing the test isolates (adjusted to 0.5 McFarland turbidity standards); and the plates were incubated at $30 \mathrm{oC}$ for $18-24$ hours. Susceptibility testing was carried out in triplicate for each isolate and the mean inhibition zone diameter (IZD) was recorded as the final IZD and reported as per the CLSI guidelines. ${ }^{11,12}$ P. aeruginosa ATCC 25668 was used as quality control strain for the antimicrobial susceptibility studies.

\section{Screening test for AmpC $\beta$-lactamase}

The presence of AmpC $\beta$-lactamase was screened for in the test $P$. aeruginosa isolates according to the method of Singhal et al. ${ }^{13}$. A suspension of the test organism (adjusted to 0.5 McFarland turbidity standards) was swabbed on Mueller-Hinton agar plates and antibiotic disk of cefotaxime $(30 \mu \mathrm{g})$ and ceftazidime $(30 \mu \mathrm{g})$ were placed adjacent to cefoxitin $(30 \mu \mathrm{g})$ disk at a distance of $20 \mathrm{~mm}$ from each other. Bacterial isolates showing blunting of ceftazidime or cefotaxime zone of inhibition adjacent to cefoxitin disk or showing reduced susceptibility to either of ceftazidime or cefotaxime and cefoxitin were considered possible AmpC $\beta$-lactamase producers.

\section{Confirmatory test for AmpC $\beta$-lactamase production}

AmpC $\beta$-lactamase production in the test isolates was phenotypically confirmed using the ceftazidime-imipenem antagonism test (CIAT) which was performed using ceftazidime $(30 \mu \mathrm{g})$ disk, cefoxitin $(30$ $\mu \mathrm{g})$ disk and imipenem disk $(10 \mu \mathrm{g})$ as described by Cantarelli et al. ${ }^{14}$ Ceftazidime disc and imipenem disk were placed at a distance of $20 \mathrm{~mm}$ apart on Mueller-Hinton agar plate previously inoculated with a suspension of the test bacteria (adjusted to 0.5 McFarland turbidity standards) that showed reduced susceptibility to cefoxitin. A cefoxitin disk $(30 \mu \mathrm{g})$ was also placed at a distance of $20 \mathrm{~mm}$ from the ceftazidime disk for comparison. All the susceptibility test plates were incubated at $30 \mathrm{oC}$ for $18-24 \mathrm{hrs}$. Antagonism indicated by a visible reduction in the inhibition zone around the ceftazidime disk adjacent to the imipenem or cefoxitin disk was inferred as a positive inducible AmpC $\beta$-lactamase production. ${ }^{11,14}$

\section{Results}

Table 1 shows the rate of recovery of $P$. aeruginosa isolates from the feacal swab samples of the feacal matter of cow. Out of the 40 feacal swab samples that was bacteriologically analyzed in this study, a total of 12 P. aeruginosa isolates was isolated on cetrimide selective agar incorporated with glycerol (Table 1). The result of antimicrobial susceptibility studies carried out on the isolated $P$. aeruginosa isolates is shown in Figure 1. The $P$. aeruginosa isolates were found to be mostly resistant to ceftriaxone (100\%), ertapenem (100\%), oxacillin $(100 \%)$, cefoxitin (100\%), amikacin $(58.3 \%)$ and cefotaxime $(75 \%)$. Meropenem, a carbapenem antibiotic also had little inhibitory activity against the $P$. aeruginosa isolates (Figure 1). However, the isolated $P$. aeruginosa isolates were found to be susceptible to the antimicrobial action of imipenem $(100 \%)$, ceftazidime $(83.3 \%)$, ofloxacin $(83.3$ $\%$ ), gentamicin $(91.7 \%$ ), and ciprofloxacin $(91.7 \%)$. Table 2 shows the frequency of AmpC $\beta$-lactamase-producing P. aeruginosa isolates phenotypically confirmed by the ceftazidime-imipenem antagonism test (CIAT). From a total of $12 P$. aeruginosa isolates that was recovered from the feacal swab samples of cow dung's, AmpC $\beta$-lactamase production was phenotypically confirmed in 3 ( 25 \%) P. aeruginosa isolates by the CIAT method. However, the other 9 isolates of $P$. aeruginosa isolates were not confirmed to produce AmpC $\beta$-lactamase as determined by the ceftazidime-imipenem antagonism test (CIAT).

Table I Recovery rate of $P$. aeruginosa on cetrimide selective agar

\begin{tabular}{lll}
\hline Sample source & No of samples & No (\%) of isolates \\
\hline Feacal matter of cow & 40 & $12(30)$ \\
\hline
\end{tabular}

Table 2 Frequency of AmpC $\beta$-lactamase producing $P$. aeruginosa isolates

\begin{tabular}{lllll}
\hline Organism & Source & $\begin{array}{l}\mathbf{n}(\%) \text { of } \\
\text { isolates }\end{array}$ & $\begin{array}{l}\text { Ampc } \\
\text { positive n (\%) }\end{array}$ & $\begin{array}{l}\text { Ampc negative } \\
\mathbf{n}(\%)\end{array}$ \\
\hline $\begin{array}{l}\text { P. } \\
\text { aeruginosa }\end{array}$ & $\begin{array}{l}\text { Feacal } \\
\text { matter of } \\
\text { cow }\end{array}$ & 12 & $3(25)$ & $9(75)$ \\
\hline
\end{tabular}

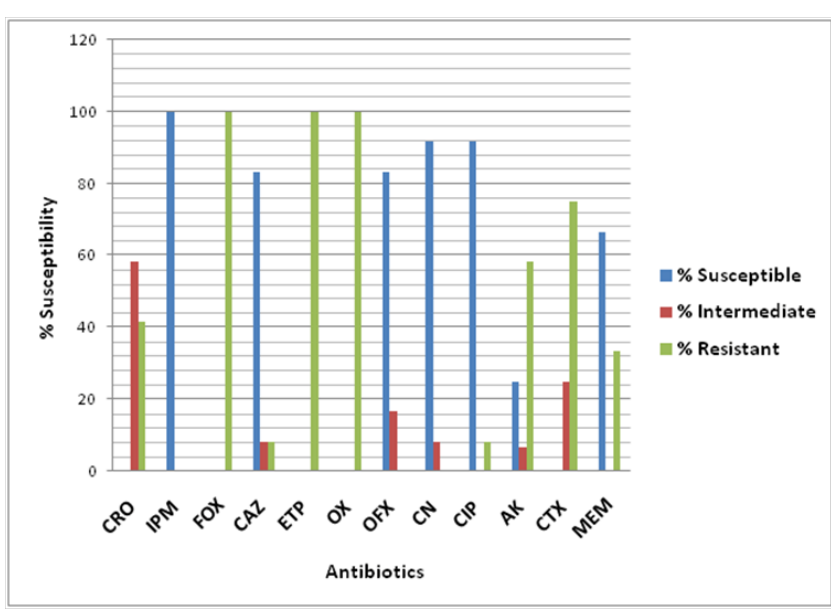

Figure I Antimicrobial susceptibility profile of the $P$. aeruginosa isolates. Key: CRO: Ceftriaxone; IPM: Imipenem; FOX: Cefoxitin; CAZ: Ceftazidime; ETP: Ertapenem; OX: Oxacillin; OFX: Ofloxacin; CN: Gentamicin; CIP: Ciprofloxacin;AK:Amikacin; CTX: Cefotaxime; MEM: Meropenem.

\section{Discussion}

The production of AmpC $\beta$-lactamases inclusive of extended spectrum $\beta$-lactamases (ESBLs) and metallo $\beta$-lactamases (MBLs) 
is one of the mechanisms of drug resistance in Gram negative bacteria especially those in the Enterobacteriaceae family and some non-enteric bacteria such as $P$. aeruginosa. Since the detection of multidrug resistant bacteria including those that produce AmpC $\beta$-lactamases is fundamental for gathering sound epidemiological data (needed for infection control measures for example) and proper patients management, this study was designed to presumptively detect the possible occurrence of AmpC-producing P. aeruginosa in feacal matter of cow from a local abattoir. In this study, the frequency of $P$. aeruginosa isolates from the 40 feacal swab samples of cow dung's analyzed was $30 \%$; and this represents a total of $12 P$. aeruginosa isolates that was isolated in this study. P. aeruginosa is a nosocomial and opportunistic Gram negative bacterium that is notorious in causing both community and hospital acquired infection/ diseases; and the bacterium is intrinsically resistant to antimicrobial agents..$^{15}$ It was also observed that the $P$. aeruginosa isolates were highly resistant to the cephamycin, cefoxitin. Ertapenem and oxacillin also had no inhibitory activity against the $P$. aeruginosa isolates. The $P$. aeruginosa isolates was also found to be highly resistant to amikacin, cefotaxime and meropenem. However, the carbapenem, imipenem showed satisfactory level of antimicrobial activity against the $P$. aeruginosa isolates from the feacal samples of animal dung. Ceftazidime, ofloxacin, ciprofloxacin and gentamicin showed appreciable level of antimicrobial activity against the $P$. aeruginosa isolates at the rate of $83.3 \%, 83.3 \%, 91.7 \%$, and 91.7 $\%$ respectively. Reports around the world show that $P$. aeruginosa is notorious for its resistance to antibiotics. ${ }^{16-18}$ Our result on the antimicrobial susceptibility patterns of $P$. aeruginosa isolates to antibiotics is similar to the work of Franco et al. ${ }^{19}$, in which high-level resistance of $P$. aeruginosa isolates to the carbapenems was reported in Brazil. Our result is also similar to the work of Aibinu et al..$^{20} \&$ Olutayo and Abimbola. ${ }^{21}$ - who reported similar resistance patterns of $P$. aeruginosa isolates in southwest Nigeria. Bashir et al. ${ }^{22}$ and Akinduti et al. ${ }^{23}$ also reported in their study carried out in Kashmir and Abeokuta respectively that $P$. aeruginosa is a multidrug resistant organism that is notoriously resistant to several antibiotic classes. And the multidrug resistant nature of $P$. aeruginosa isolates has been confirmed by Fernandez et al. ${ }^{16}$ in the Americas as well. The prevalence of AmpC $\beta$-lactamase positive $P$. aeruginosa isolates was $25 \%$. This rate of prevalence of AmpC-producing P. aeruginosa isolates is similar to the work of Akinduti et al. ${ }^{23}$ carried out in southwest Nigeria. The prevalence of AmpC-positive P. aeruginosa isolates in this study is lower than the report of Abd El-Baky et al. ${ }^{24}$ who reported higher prevalence of AmpC-positive P. aeruginosa (72.4 $\%)$ in their study. The occurrence of antibiotic resistant bacteria from the community portend public health risk to mankind owing to the fact that drug resistant bacteria could easily be transmitted in human population through the food chain since antibiotics now serves as an important factor in the rearing and production of livestock and even poultry birds. Conclusively, proper surveillance measures aimed at detecting and taming this menace is critical to bring the emergence and spread of AmpC $\beta$-lactamase-producing $P$. aeruginosa in the community under control.

\section{Conflicts of interest}

There is no conflict of interest.

\section{Acknowledgements}

None.

\section{Funding}

None.

\section{References}

1. Perez perez FJ, Hanson ND. Detection of Plasmid - Mediated Amp C beta - lactamase genes in clinical isolates by using multiplex PCR. $J$ Clin Microbiol. 2002;40(6):2153-2162.

2. Jacoby GA, Munoz price LS. Mechanism of disease: The New $\beta$ lactamases. New England Journal of Medicine. 2005;352(4):380-391.

3. Jacoby GA. AmpC $\beta$-lactamases. Clinical Microbiology Reviews. 2009;22(1):161-182.

4. Hossain A, Reisbig MD, Hanson ND. Plasmid-encoded functions compensate for the biological cost of $\mathrm{AmpC}$ overexpression in a clinical isolate of Salmonella typhimurium. J Antimicrob Chemother. 2004;53(6):964-970.

5. Hemalatha V, Padma M, Sekar U, et al. Detection of AmpC beta lactamases production in Escherichia coli and Klebsiellaby an inhibitor based method. Indian J Med Res. 2007;126(3):220-223.

6. Bush K, Jacoby GA, Medeiros AA. A Functional Classification of Scheme for $\beta$-Lactamases and its Correlation to Molecular Structure. Antimicrob Agents Chemother. 1995;39(6):1211-1233.

7. Ejikeugwu C, Esimone C, Iroha I, et al. Phenotypic detection of AmpC beta-lactamase among anal Pseudomonas aeruginosa isolates in a Nigerian abattoir. Arch Clin Microbiol. 2016;7(2):1-5.

8. Choi SH, Lee JE, Park SJ, et al. Emergence of antibiotic resistance during therapy for infections caused by Enterobacteriaceae producing AmpC $\beta$-lactamase: implications for antibiotic use. Antimicrob Agents Chemother. 2008;52(3):995-1000.

9. Cheesbrough M. District Laboratory Practice in Tropical Countries. ( $2^{\text {nd }}$ edn), Cambridge University Press, UK. 2006;178-187.

10. Baron E.J, Peterson L.R, Finegold SM. Nonfermentative Gram negative bacilli and coccobacilli. Bailey and Scott's Diagnostic Microbiology ( $9^{\text {th }}$ edn) St. Louis, USA. 1994;386-405.

11. Ejikeugwu C, Duru C, Iroha I, et al. Detection of Escherichia coli strains producing AmpC enzymes using Ceftazidime-Imipenem Antagonism Test (CIAT). International Journal of Research Studies in Biosciences. 2017;5(1):41-45.

12. Clinical Laboratory Standard Institute, CLSI. Performance standards for antimicrobial disk susceptibility test. Fifteenth informational supplement, CLSI document M100-S15. Wayne, PA. USA. 2011.

13. Singhal S, Mathur T, Khan S, et al. Evaluation of Methods for AmpC Beta-Lactamase in Gram Negative Clinical Isolates from Tertiary Care Hospitals. Indian J Med Microbiol. 2005;23(2):120-124.

14. Cantarelli VV, Inamine E, Brodt TCZ, et al. Utility of the ceftazidimeimipenem antagonism test (CIAT) to detect and confirm the presence of inducible AmpC beta-lactamases among Enterobacteriaceae. Braz JInfect Dis. 2007;11(2):237-239.

15. Brooks GF, Butel JS, Morse SA. Medical Microbiology (23rd edn) McGraw Hill Publishers, USA. 2004;48-260.

16. Fernandez M, Conde S, Torre J, et al. Mechanisms of resistance to chloramphenicol in Pseudomonas putida KT2440. Antimicrob Agents Chemother. 2012;56(2):1001-1009.

17. Walsh TR, Toleman MA, Poirel L, et al. Metallo $\beta$-Lactamases: The Quiet Before the Storm? Clin Microbiol Rev. 2005;18(2):306-325.

18. Wegener HC. Antibiotics in animal feed and their role in resistance development. Curr Opin Microbiol. 2003;6(5):439-445. 
19. Fischer J, Rodriguez I, Schmoger S, et al. Escherichia coli producing VIM-1 carbapenemase isolated on a pig farm. J Antimicrob Chemother. 2012;67(7):1793-1795.

20. Aibinu I, Nwanneka T, Odugbemi T. Occurrence of ESBLs and MBL in Clinical Isolates of Pseudomonas aeruginosa from Lagos, Nigeria. Journal of American Science. 2007;3(4):81-85.

21. Olutayo IF, Abimbola OA. Antibiogram of Escherichia coli and Pseudomonas strains isolated from wastewater generated by an abattoir as it journeys into a receiving river. Advances in Microbiology. 2016;6:303-309.
22. Bashir D, Thokar MA, Fomda BA, et al. Detection of metallo- betalactamase (MBL) producing Pseudomonas aeruginosa at a tertiary care hospital in Kashmir. African Journal of Microbiology Research. 2011;5(2):164-172.

23. Akinduti PA, Ejilude O, Motayo BO, et al. Emerging multidrug resistant AmpC beta-lactamase and carbapenemase enteric isolates in Abeokuta, Nigeria. Nature and Science. 2012;10(7):70-74.

24. Abd El Baky RM, Abd El Azeim NH, Gad GFM. Prevalence of extended spectrum beta lactamase, AmpC beta lactamase, and metallo beta lactamase among clinical isolates of Pseudomonas aeruginosa. Journal of Advanced Biotechnology and Bioengineering. 2013;1:22-29. 